Roger Keller Celeste

Paulo Nadanovsky

Antonio Ponce De Leon
Departamento de Epidemiologia. Instituto de Medicina Social. Universidade do Estado do Rio de Janeiro. Rio de Janeiro, RJ, Brasil.

Correspondência | Correspondence: Roger Keller Celeste

R. São Francisco Xavier, 524

$7^{\circ}$ andar, blocos $\mathrm{D}$ e $\mathrm{E}$

20550-90 Rio de Janeiro, RJ, Brasil

E-mail: rk_celeste@hotmail.com

\section{Associação entre procedimentos preventivos no serviço público de odontologia e a prevalência de cárie dentária}

\section{Association between preventive care provided in public dental services and caries prevalence}

\section{RESUMO}

OBJETIVO: Avaliar a associação entre as atividades odontológicas preventivas do serviço público de odontologia e a saúde bucal de jovens.

MÉTODOS: Foram utilizados dados de 4.033 jovens de 15 a 19 anos, de 85 municípios do estado do Rio Grande do Sul, obtidos no inquérito epidemiológico Saúde Bucal Brasil, realizado no período de 2002-2003. As variáveis estudadas foram: idade, sexo renda, escolaridade, tempo desde a última visita ao dentista, motivo da visita, e presença de flúor na água de abastecimento. Dados sobre as atividades odontológicas foram extraídos da base de dados do Sistema de Informações Ambulatoriais do Sistema Único de Saúde. Para análise estatística foi utilizada a regressão logística multinível.

RESULTADOS: Os indivíduos dos 21 municípios com menores taxas de procedimentos (por 100 habitantes) de procedimentos individuais preventivos (limpeza + flúor + selante) tiveram 2,27 (IC 95\%: 1,45;3,56) vezes mais chance de ter uma cárie não restaurada do que residentes dos 21 municípios com maiores taxas. Essa chance permaneceu mesmo depois de ajustada por fatores individuais e contextuais, mas decresceu para 1,76 (IC 95\%: 1,13;2,72). A proporção da variabilidade atribuível aos municípios foi de $14,1 \%$ para o modelo vazio e $10,5 \%$ para o modelo cheio.

CONCLUSÕES: O serviço público de odontologia no Rio Grande do Sul pode ter contribuído para a redução no número de cáries não restauradas em jovens. Porém, não foi possível detectar influência desse serviço na experiência total da cárie.

DESCRITORES: Cárie dentária, epidemiologia. Cárie dentária, prevenção e controle. Serviços de saúde bucal. Avaliação de serviços de saúde. Fatores socioeconômicos. 


\section{ABSTRACT}

OBJECTIVE: To assess the association between preventive care provided in public dental services and young people's oral health.

METHODS: Oral health data on 4,033 young people aged 15 to 19 years living in 85 municipalities of the state of Rio Grande do Sul, Southern Brazil, were obtained from the national oral health survey "Saúde Bucal Brasil 2003" for the period 2002-2003. The following variables were studied: age, gender, income, education, time elapsed since last dental visit, reason for dental visit, and water fluoridation. Data on dental care services were obtained from the national database of public health services. Statistical analysis was performed using multilevel logistic regression.

RESULTS: Youngsters from the 21 municipalities with the lowest preventive care (scaling + fluoride + sealants) rates per 100 inhabitants were $2.27(95 \%$ CI: $1.45 ; 3.56)$ more likely to have non-filled dental cavities than those from the 21 municipalities with the highest care rates. After adjustment for a number of individual and contextual factors this likelihood decreased to $1.76(95 \% \mathrm{CI}$ : $1.13 ; 2.72)$. The variance attributable to variables at municipal level was $14.1 \%$ for the empty model and decreased to $10.5 \%$ for the fully adjusted model.

CONCLUSIONS: Rio Grande do Sul public dental services may have contributed for the reduction in the number of non-filled cavities in young people. However, it was not possible to detect the impact of this service on total dental caries experience.

KEY WORDS: Dental caries, epidemiology. Dental caries, prevention \& control. Dental health services. Health services evaluation. Socioeconomic factors.

\section{INTRODUÇÃO}

O impacto dos serviços de saúde na prevenção de doenças ainda é controverso. Defensores de que o papel dos serviços é importante argumentam que o acesso a tratamentos eficazes pode reduzir o nível de doenças. ${ }^{3}$ Entretanto, deve-se considerar que a eficácia de intervenções preventivas, mesmo quando comprovada em ensaios clínicos controlados sob condições ideais, nem sempre é confirmada no dia-a-dia dos serviços, sob condições reais. ${ }^{16,21}$

Somente 3\% da variação no declínio da cárie entre as décadas de 1970 e 1980 em países desenvolvidos foram atribuídos aos serviços odontológicos, enquanto que $65 \%$ foram explicados por melhorias amplas nas condições socioeconômicas. ${ }^{14}$ Sucessivos tratamentos restauradores são indicador de acesso aos serviços odontológicos, mas incapazes de evitar a perda do dente em muitos casos. ${ }^{5}$ Todavia, visitar o dentista periodicamente para revisão de rotina parece reduzir o risco de perder dentes. ${ }^{6}$ Com relação à cárie dentária de forma geral, o serviço odontológico parece reduzir o número de dentes perdidos e o número de dentes com cáries não restauradas e aumentar o número de dentes com restaurações. ${ }^{7}$ A experiência total de cárie, i.e., a soma do número de dentes cariados, perdidos e obturados, parece não ser alterada pelos serviços odontológicos. ${ }^{7}$ Aumento no número de visitas ao dentista foi correlacionado com uma mudança para melhor na saúde bucal auto-referida, mas tal melhora não foi atribuída aos procedimentos preventivos. ${ }^{11}$

A diretriz* atual para os serviços públicos odontológicos brasileiros tem como pressuposto harmonizar ações preventivas com as da assistência, além de "acompanhar o impacto das ações de saúde bucal por meio de indicadores adequados...". Essa diretriz aponta pelo menos dois caminhos: verificar tendências na utilização dos serviços odontológicos pela população que deles potencialmente pode se beneficiar (avaliação do processo), ou avaliar o efeito da utilização desses serviços na saúde bucal dos usuários em potencial (avaliação do resultado). A avaliação do resultado deve idealmente

* Ministério da Saúde. Secretaria de Atenção à Saúde. Departamento de Atenção Básica. Coordenação Nacional de Saúde Bucal. Diretrizes da política nacional de saúde bucal. Brasília; 2004. p.16. [Acesso em 26/7/2006] Disponível em: http://dtr2004.saude.gov.br/dab/saudebucal/publicacoes/diretrizes_da_politica_nacional_de_saude_bucal.pdf 
basear-se em estudos experimentais controlados, principalmente grandes ensaios clínicos, porém estudos observacionais são mais exeqüíveis. Em estudos não experimentais, quando não se encontra uma associação entre indicadores de processo e de resultado, pode-se concluir que o serviço não foi efetivo. Porém, quando uma associação positiva está presente, não é possível concluir que o serviço foi efetivo. Nesse caso há várias explicações alternativas que devem ser excluídas.

Poucos estudos foram realizados ligando indicadores de processo dos serviços de saúde pública com indicadores de resultados desses serviços. ${ }^{10}$

O objetivo do presente estudo foi avaliar a associação entre as atividades de prevenção da cárie dentária, realizadas pelos dentistas dos serviços públicos de odontologia, e a prevalência de cárie em uma população de usuários em potencial desses serviços.

\section{MÉTODOS}

Foram utilizados dados transversais de duas fontes. Os dados de cárie dentária, socioeconômicos, demográficos e auto-relato da utilização de serviços odontológicos foram obtidos de um inquérito epidemiológico iniciado no estado do Rio Grande do Sul, no ano de 2002 e concluído em 2003. Tal inquérito foi realizado segundo as recomendações da Organização Mundial de Saúde. Dados sobre os procedimentos odontológicos realizados pelos dentistas que trabalham na rede pública municipal foram obtidos do Datasus.

O banco de dados epidemiológicos foi fornecido pela Seção de Saúde Bucal do Departamento de Ações em Saúde da Secretaria de Saúde de Estado do Rio Grande do Sul, entidade coordenadora do mencionado inquérito (SBGaúcho). Nele estavam incluídos 34.472 indivíduos de uma amostra de 95 municípios, representando uma expansão da amostra inicial do inquérito maior, o SBBrasil.

Foram selecionadas para análise apenas as informações das pessoas que relataram ter ido ao dentista em até três anos antes da coleta de dados, em alguma unidade de saúde pública do Sistema Único de Saúde (SUS) e que não fossem edêntulas.

Apenas os dados sobre cárie dental na faixa etária de 1519 anos foram utilizados. O índice de dentes cariados, perdidos e obturados (CPOD) foi dicotomizado, obtendo-se o percentual de indivíduos com ao menos um dente cariado, perdido ou obturado (CPOD $>0$ ). Cada componente do índice CPOD foi também analisado individualmente da mesma forma $(\mathrm{C}>0, \mathrm{P}>0$ e $\mathrm{O}>0)$. Os exames foram realizados por dentistas dos serviços de saúde de cada município em visitas domiciliares, após um período de treinamento.
No SBGaúcho foram também coletados dados por meio de um questionário individual, contendo as variáveis demográficas, sexo e idade e socioeconômicas: escolaridade e renda familiar per capita em salários mínimos (SM) do ano de 2002 (à época, R \$200 UUS\$ 100). Das variáveis relativas ao uso dos serviços de saúde, foram utilizados: o tempo desde a última consulta (menos de um ano/ 1 a 2 anos) e o motivo para a consulta odontológica (manutenção/outro motivo). O SBGaúcho também apresentava dados sobre a fluoretação da água de abastecimento municipal.

Os dados sobre o percentual de moradores com abastecimento de água da rede geral canalizada em pelo menos um cômodo ou só na propriedade/terreno, foram obtidos do censo do Instituto Brasileiro de Geografia e Estatística (IBGE). Não foi considerado o abastecimento de água por outra fonte, para o ano de 2000 .

Os dados sobre os procedimentos e tamanho populacional, segundo censo do IBGE de 2000 e estimativas para 2001 e 2002 foram extraídos da base de dados do Sistema de Informações Ambulatoriais do SUS (SIA/SUS).

Para cada um dos municípios foram extraídos os dados dos anos de 2000, 2001 e 2002 contendo o número de cada procedimento (quantidade aprovada) da tabela SIA/SUS, tanto para a atenção básica odontológica como atenção especializada odontológica. Posteriormente, foram criados os seguintes indicadores:

- Taxa de procedimentos coletivos por 100 crianças (TC) - razão entre o total de procedimentos coletivos (código 0301101) pela população coberta, ou seja, pelo total de crianças residentes entre zero a 14 anos, multiplicado por 100. Procedimentos coletivos abrangem bochechos fluoretados, escovação supervisionada e atividades educativas.

- Taxa de restaurações por 100 habitantes (TR) - razão entre o total de restaurações (soma dos 12 códigos de restauração) pelo total de pessoas residentes no município no referido ano, multiplicado por 100 .

- Taxa de exodontias por 100 habitantes (TEX) - razão entre o total de exodontias simples (códigos 0304101, 0304102 e 0304103) e múltiplas (código 1005114) pelo total de pessoas residentes no município no referido ano, multiplicado por 100.

- Taxa de procedimentos individuais preventivos por 100 habitantes (TP) - soma do número de procedimentos preventivos (sessão de aplicação terapêutica intensiva de flúor, código-0302201; aplicação de cariostático por dente, código-0302202; aplicação de selante por dente, código-0302203; sessão de 
controle da placa bacteriana, código-0302204; escariação por dente, código-0302205), dividido pelo total de pessoas residentes no município no referido ano, multiplicado por 100 .

Os valores das taxas utilizadas nas análises foram as médias dos anos de 2000 a 2002, para evitar que um ano atípico pudesse influenciar os resultados. Esses foram os anos imediatamente antecedentes aos dados sobre a cárie dentária.

As taxas TC, TR, TEX e TP foram correlacionadas aos seguintes percentuais de indivíduos: com índice CPOD $>0$; com ao menos uma cárie não restaurada $(\mathrm{C}>0)$; com ao menos uma restauração $(\mathrm{O}>0)$; com ao menos um dente perdido por cárie $(\mathrm{P}>0)$. As análises foram realizadas no programa Stata 9.01.

A magnitude do efeito da taxa TP foi avaliada por meio de regressão logística multinível com intercepto aleatório. $\mathrm{O}$ desfecho foi ter ao menos uma cárie não restaurada. Os indivíduos foram a unidade de primeiro nível de análise; agrupados nos seus respectivos municípios, formaram unidades de segundo nível.

A partir do modelo multinível foi particionada a variância total nos dois níveis, segundo o método D apresentado por Goldstein et $\mathrm{al}^{9}$ (2002). A proporção da variância explicada foi calculada segundo fórmula descrita em Snijders \& Bosker ${ }^{18}$ (1999), utilizando- se estimadores de quasi-verosimilhança de segunda ordem (Penalized Quasi-likelihood). As diferenças de prevalências ajustadas entre municípios foram testadas utilizando o teste de qui-quadrado. A análise estatística multinível foi realizada no programa MlwiN 2.02.

\section{RESULTADOS}

Dos 95 municípios inicialmente selecionados, dez foram excluídos porque não coletaram dados, ou porque a coleta apresentou problemas. Depois de aplicados os critérios de exclusão, a análise procedeu com 4.033 indivíduos no primeiro nível e 85 municípios no segundo nível. O número de indivíduos por município variou entre 9 a 106, com média de 47,4 indivíduos por município.

O CPOD médio na amostra dos jovens de 15-19 anos usuários dos serviços públicos foi de 7,1 com desviopadrão (dp) de $\pm 5,1(\mathrm{C}=2 \pm 2,8 ; \mathrm{P}=1 \pm 2 ; \mathrm{O}=4,1 \pm 4)$.

A prevalência de CPOD $>0$ foi de $92,7 \%$ com intervalo de confiança de 95\% (IC 95\%) de 91,9\%;93,5\%. A prevalência de C $>0$ foi de 56,9\% (IC 95\%: 55,4;58,4), de $\mathrm{P}>0$ foi de $39,3 \%$ (IC 95\%: 37,8;40,8) e de $\mathrm{O}>0$ foi de $75,8 \%$ (IC 95\%: 74,5;77,1).

A maioria dos municípios teve taxa relativamente baixa de procedimentos odontológicos. A média de procedimentos preventivos foi de 11,2/100 habitantes

Tabela 1. Taxas dos principais procedimentos odontológicos ambulatoriais realizados nos serviços públicos municipais. Estado do Rio Grande do Sul, 2000-2002.

\begin{tabular}{ccccc}
\hline Procedimento & Média/100 hab (DP) & Mínimo & Mediana & Máximo \\
\hline Coletivos* & $87,5(161,6)$ & 0 & 15,4 & 745,0 \\
Preventivos & $11,2(13,4)$ & 0 & 6,9 & 66,6 \\
Quartil 1 & $1,3(1,1)$ & 0 & 1,5 & 3,1 \\
Quartil 2 & $4,7(1,0)$ & 3,1 & 4,4 & 6,5 \\
Quartil 3 & $9,2(1,5)$ & 6,9 & 9,4 & 11,8 \\
Quartil 4 & $28,9(15,6)$ & 11,9 & 24,3 & 66,6 \\
Exodontias & $7,8(4,4)$ & 0 & 7,0 & 20,5 \\
Restaurações & $19,5(14,9)$ & 0 & 16,3 & 89,2 \\
\hline
\end{tabular}

* taxa calculada a partir da população de 0 a 14 anos.

Tabela 2. Correlação entre as prevalências de dentes cariados, perdidos e obturados em jovens de 15 a 19 anos de idade e procedimentos. Estado do Rio Grande do Sul, 2000-2002.

\begin{tabular}{lcccc}
\hline & \multicolumn{2}{c}{ Desfecho } \\
Procedimento & $\%$ CPOD $>0$ & $\%$ P $>0$ & $\%$ C $>0$ & $\%$ O $>0$ \\
\hline Exodontias & $0,30^{*}$ & $0,33^{*}$ & 0,18 & 0,18 \\
Restaurações & $0,40^{*}$ & 0,16 & $-0,17$ & $0,67^{*}$ \\
Procedimentos preventivos & $-0,02$ & $-0,08$ & $-0,33^{*}$ & $0,39^{*}$ \\
Procedimentos coletivos & $-0,24$ & $-0,17$ & $-0,13$ & $-0,04$ \\
\hline
\end{tabular}

${ }^{*} \mathrm{p}<0,01$; correlação de Spearman. 
Tabela 3. Razão de chances da presença de cárie não restaurada (C>0) em jovens de 15 a 19 anos de idade, de acordo com serviços odontológicos e variáveis sociodemgráficas e de saúde bucal. Estado do Rio Grande do Sul, 2000-2002.

\begin{tabular}{|c|c|c|c|}
\hline Variável & $\begin{array}{l}\text { OR bruto } \\
\text { (IC 95\%) }\end{array}$ & $\begin{array}{l}\text { OR ajustado } \\
\text { (IC } 95 \%)^{*}\end{array}$ & $\mathrm{~N}(\%)$ \\
\hline \multicolumn{4}{|l|}{ Atividade dos serviços odontológicos } \\
\hline $\begin{array}{l}\text { Taxa de procedimentos preventivos indivi- } \\
\text { dualizados por } 100 \text { habitantes }\end{array}$ & & & $85(100)$ \\
\hline Quartil 4 & 1 & 1 & $22(25,9)$ \\
\hline Quartil 3 & $1,61(1,03 ; 2,52)$ & $1,47(0,97 ; 2,23)$ & $21(24,7)$ \\
\hline Quartil 2 & $1,61(1,03 ; 2,51)$ & $1,43(0,93 ; 2,20)$ & $21(24,7)$ \\
\hline Quartil 1 & $2,27(1,45 ; 3,56)$ & $1,76(1,13 ; 2,72)$ & $21(24,7)$ \\
\hline Taxa de exodontias por 100 habitantes & & $1,03(0,99 ; 1,07)$ & $85(100)$ \\
\hline Taxa de restaurações por 100 habitantes & & $0,99(0,98 ; 1,00)$ & $85(100)$ \\
\hline Sexo & & & $4033(100)$ \\
\hline Feminino & & 1 & $2413(59,8)$ \\
\hline Masculino & & $1,45(1,26 ; 1,67)$ & $1620(40,2)$ \\
\hline Idade (anos) & & $1,1(1,06 ; 1,17)$ & $4033(100)$ \\
\hline Escolaridade & & & $4033(100)$ \\
\hline Médio completo & & 1 & $290(7,2)$ \\
\hline Médio incompleto & & $1,27(0,95 ; 1,70)$ & $1822(45,2)$ \\
\hline Fundamental completo & & $2,14(1,58 ; 2,92)$ & $1698(42,1)$ \\
\hline Analfabeto ou fundamental incompleto & & $3,52(2,26 ; 5,48)$ & $223(5,5)$ \\
\hline Renda familiar per capita (SM) & & & $4033(100)$ \\
\hline$>2 \mathrm{SM}$ & & 1 & $70(1,7)$ \\
\hline 1 a 2 SM & & $1,97(1,09 ; 3,56)$ & $443(11)$ \\
\hline $1 / 2$ a $1 \mathrm{SM}$ & & $2,16(1,22 ; 3,80)$ & $1127(27,9)$ \\
\hline$<1 / 2 \mathrm{SM}$ & & $2,60(1,48 ; 4,57)$ & $2393(59,3)$ \\
\hline Motivo da última visita ao dentista & & & $4033(100)$ \\
\hline Manutenção & & 1 & $1730(42,9)$ \\
\hline Outro motivo & & $2,86(2,46 ; 3,32)$ & $2303(57,1)$ \\
\hline Tempo desde a última visita ao dentista & & & $4033(100)$ \\
\hline$<1$ ano & & 1 & $3026(75)$ \\
\hline 1 a 2 anos & & $1,13(0,96 ; 1,33)$ & $1007(25)$ \\
\hline Fluoretação da água de abastecimento & & & $85(100)$ \\
\hline Sim & & 1 & $52(61,2)$ \\
\hline Não & & $1,18(0,82 ; 1,69)$ & $33(38,8)$ \\
\hline Percentual de Moradores com água encanada** & & $0,99(0,98 ; 1,00)$ & $85(100)$ \\
\hline
\end{tabular}

Nota: Variáveis individuais $\mathrm{N}=4.033$; variáveis municipais $\mathrm{N}=85$.

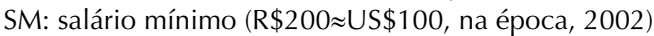

* Valores ajustados para todas a variáveis da coluna

** Intervalo de confiança obtido por arredondamento

em todos os municípios e o quartil de municípios com maior quantidade de procedimentos preventivos apresentaram, em média, 27,3 procedimentos a mais do que o quartil com menor quantidade (Tabela 1).

Municípios com maiores taxas de exodontias apresentaram maiores prevalências de $\mathrm{P}>0$ e de $\mathrm{CPOD}>0$. Não houve correlação entre a taxa de exodontia e as prevalências de $\mathrm{C}>0$ e de $\mathrm{O}>0$. Maiores taxas de realização de restaurações foram encontradas em municípios com maiores prevalências de $\mathrm{O}>0$ e de $\mathrm{CPOD}>0$, e não houve correlação com as prevalências de $\mathrm{P}>0$ e $\mathrm{C}>0$. A taxa de procedimentos preventivos individualizados apresentou correlação com a prevalência de $\mathrm{C}>0$ (negativa) e de $\mathrm{O}>0$ (positiva), mas não com a prevalência de $\mathrm{P}>0$ e de CPOD $>0$. A correlação da taxa de restaurações 
Tabela 4. Coeficientes de partição de variância referente ao nível municipal e proporção explicada (R2) da variabilidade entre indivíduos com ao menos uma cárie não restaurada por diferentes modelos logísticos. Estado do Rio Grande do Sul, 2000-2002.

\begin{tabular}{lcc}
\hline Modelo & R2 & VPC \\
\hline Modelo vazio & $0,0 \%$ & $14,1 \%$ \\
Modelo 1* & $8,8 \%$ & $12,3 \%$ \\
Modelo 1 + serviços** & $11,2 \%$ & $11,6 \%$ \\
Modelo 1 + condições & $14,1 \%$ & $11,6 \%$ \\
materiais*** & & \\
Modelo 1 + serviços + & $16,8 \%$ & $10,5 \%$ \\
Condições materiais & &
\end{tabular}

* Variáveis incluídas: sexo, idade, motivo da última visita.

** Variáveis dos serviços: taxa preventiva, taxa de exodontias, taxa de restaurações e tempo desde a última consulta.

*** Condições materiais e sociais de vida: escolaridade, renda, água fluoretada e água encanada.

VPC - Coeficiente de partição de variância

realizadas com a prevalência de $\mathrm{O}>0$ foi maior $(0,67)$ do que a correlação da taxa de procedimentos preventivos individualizados com a prevalência de $\mathrm{O}>0(0,39)$. A taxa de procedimentos coletivos não apresentou correlação com nenhum indicador de prevalência de cárie (Tabela 2).

Houve forte correlação entre taxas municipais de procedimentos clínicos (soma de todos os procedimentos) e taxas de restaurações $\left(r_{s}=0,81 \mathrm{p}<0,01\right)$. A taxa de procedimentos preventivos teve correlação moderada $\left(r_{s}=0,32 p<0,01\right)$ com a taxa de restaurações, mas não com a taxa de exodontias $\left(\mathrm{r}_{\mathrm{s}}=0,02 \mathrm{p}<0,81\right)$.

As chances de um jovem apresentar ao menos uma cárie não restaurada, residindo nos 21 municípios com menores taxas de procedimentos preventivos individualizados ( $1^{\circ}$ quartil), foram 2,27 vezes maiores do que de jovens residindo nos 21 municípios com maiores taxas ( $4^{\circ}$ quartil). Essas chances permaneceram estatisticamente significantes mesmo depois de ajustadas pelas variáveis da Tabela 2, mas decresceram para 1,76. Além da presença de menores taxas de procedimentos preventivos individualizados, a chance de existir cárie não restaurada aumentou para pessoas do sexo masculino, escolaridade e renda baixas e que relataram que a última visita ao dentista não foi para manutenção. As taxas de exodontias (TEX) e de restaurações (TR) não alteraram as chances de pessoas apresentarem cárie não restaurada, ou de municípios apresentarem maiores prevalências de $\mathrm{C}>0$. Água encanada e fluoretada também não influenciaram as chances e prevalências de $\mathrm{C}>0$ (Tabela 3).

A Tabela 4 apresenta os percentuais da variância explicada $\left(\mathrm{R}^{2}\right)$ por diferentes modelos e o percentual da variância não explicada atribuível a diferenças entre os municípios (Coeficiente de partição de variância
- VPC). O modelo cheio explicou $16,8 \%$ da variação na presença de cáries não restauradas entre os indivíduos. Nesse modelo, $10,5 \%$ da variabilidade não explicada é atribuível a diferenças entre características das unidades do segundo nível de análise (diferenças entre os municípios). As variáveis que representaram as condições materiais de vida tiveram maior poder explicativo do que as que representaram a atividade dos serviços odontológicos. Entretanto, a metodologia utilizada para tal cálculo não permite o uso de testes de hipótese, de modo que a diferença de $\mathrm{R}^{2}$ observada pode não ser estatisticamente significante. Além disso, os valores de $\mathrm{R}^{2}$ não foram ajustados pelo número de variáveis no modelo. As variáveis explicativas não conseguiram remover a diferença estatisticamente significante entre as unidades de segundo nível (os municípios). Para isso foi aplicado o teste do qui-quadrado para significância do intercepto aleatório $\left(X^{2}=24,7 \mathrm{com} \mathrm{gl}=1, \mathrm{p}<0,001\right)$.

O diagnóstico dos resíduos para modelos multiníveis deve ser efetuado em ambos níveis de análise. Tanto os resíduos de primeiro como de segundo nível mostraram distribuição normal. Em nenhum nível houve a presença de outliers. Análises visuais dos gráficos de correlação dos resíduos com as variáveis explicativas do modelo cheio não sugeriram problemas.

\section{DISCUSSÃO}

A avaliação de desempenho i.e., de resultados, é considerada uma importante parte da avaliação dos serviços e sistemas de saúde ${ }^{2,10}$ Ainda assim, há autores que argumentam que a avaliação deveria focar mais no processo e satisfação dos usuários ${ }^{2}$ dadas as evidências de reduzida capacidade dos serviços em obter resultados que expressem melhoria no quadro epidemiológico. ${ }^{14,15,17}$ Um bom serviço seria aquele que adota procedimentos cuja eficácia clínica seja comprovada. Entretanto, o benefício oriundo desses procedimentos nem sempre será visto, pois depende da performance dos profissionais que os executam e das circunstâncias de vida das populações atendidas. Os procedimentos preventivos estudados apresentaram evidência de eficácia clínica, como o uso dos fluoretos (gel e vernizes) ${ }^{13}$ e selantes. ${ }^{1}$ Além disso, diferenças no desempenho do operador não devem influenciar a eficácia dos procedimentos, considerando-se a facilidade de execução.

O principal objetivo de uma avaliação é auxiliar gestores na tomada de decisões. Uma opção seriam os "estudos de plausibilidade", onde devem ser excluídas explicações alternativas para os achados, de forma que ao final fosse plausível que o efeito observado fosse atribuído aos serviços. ${ }^{16}$ Variáveis agregadas podem ser utilizadas para avaliação de intervenções populacionais, como realizado no presente estudo, sendo a variável ecológica uma proxy para o nível individual. A presença de falácia ecológica é discutível, pois o interesse não 
é estabelecer relação causal, uma vez que esta já foi estabelecida no nível individual em outros estudos.

Uma revisão sobre a freqüência de ida ao dentista encontrou em 14 de 18 estudos associação significante na redução do número de cavidades de cárie em indivíduos e crianças que tinham maior freqüência de visitas. ${ }^{7}$ É possível que os pacientes com maior freqüência tenham recebido mais prevenção e restaurações ou mesmo tivessem outros hábitos saudáveis. Restaurações e prevenção parecem ser eventos concomitantes. Estudo na Inglaterra mostrou que os pacientes com cavidades de cárie, que se apresentavam aos dentistas, recebiam mais procedimentos preventivos do que os pacientes de manutenção. ${ }^{20}$ Isso poderia explicar a correlação ecológica entre procedimentos preventivos e percentuais de dentes restaurados. No presente estudo, o efeito preventivo permaneceu após o controle pelo tempo desde a última visita e pela taxa de restaurações de cada município. Além disso, mesmo dentre indivíduos que realizaram consulta para check-up, aqueles que residiam em municípios com mais procedimentos preventivos apresentaram menos chances de ter cavidades, o que sugere um efeito positivo do serviço, além dos hábitos dos indivíduos.

Em concordância com outros estudos, não foi encontrada associação entre acesso aos serviços odontológicos e o índice CPOD, nem entre procedimentos preventivos e o índice CPOD. ${ }^{4,7,12,14}$ Uma possível explicação para o fato de terem sido encontradas mais cavidades não restauradas em municípios com menos atividades preventivas, mas sem associação dessas atividades com a experiência total de cárie (CPOD), é que este último pode ter englobado um acúmulo de diagnósticos falso-positivos, refletido em um aumento nos componentes perdido e obturado, desvinculado da atividade de cárie. ${ }^{17}$ Dessa forma, superfícies que a princípio não foram restauradas devido ao efeito positivo das atividades preventivas, evitando a cárie, teriam sido subseqüentemente restauradas desnecessariamente.

Outra questão é a possibilidade dos serviços odontológicos influenciarem a variação na saúde bucal entre indivíduos e entre populações. De forma geral, a influência dos serviços é aparentemente menor do que a influência das condições socioeconômicas. Os resultados encontrados agregaram novas informações a esse conhecimento.

Um aspecto a ser considerado quando se analisa o quanto um modelo estatístico explica um desfecho é o tipo de desfecho. Quando ele está no nível individual, os valores de $\mathrm{R}^{2}$ costumaram ser mais baixos. O'Brien et al apud Watt \& Sheiham ${ }^{22}$ (1999) apresentaram valores de $7 \%$ a $8 \%$ para modelos que explicaram a variabilidade de cavidades de cárie em dentição decídua e Tellez et al $^{19}$ (2006) apresentam valores de $14 \%$ para o desfecho cárie não tratada em indivíduos adultos.
Quando a variável desfecho for do tipo ecológica, os valores de $\mathrm{R}^{2}$ são mais altos. Por exemplo, Nadanovsky $\&$ Sheiham ${ }^{14}$ (1995) encontraram valores de até 65\%, Ekstrand et $\mathrm{al}^{8}$ (2003) relatam valores de até 54\% e Lucas et $\mathrm{al}^{12}$ (2005) valores de $39 \%$. Uma segunda questão é o próprio desfecho em si. Enquanto Nadanovsky \& Sheiham $^{14}$ (1995) estudaram o percentual de redução no CPOD, Ekstrand et $\mathrm{al}^{8}$ (2003) e Lucas et $\mathrm{al}^{12}$ (2005) analisaram o CPOD médio. Um terceiro ponto referese às variáveis explicativas utilizadas nos modelos de regressão múltipla. Apesar de semelhantes, raramente as variáveis foram as mesmas.

No presente estudo, o desfecho para a regressão foi apenas o componente $\mathrm{C}$ do CPOD, em nível individual, portanto, comparações com outros estudos devem ser realizadas com precaução. Uma vantagem dos modelos multiníveis é a possibilidade de partição da variância. Em modelos ecológicos, toda variabilidade entre municípios seria atribuída às características municipais, quando em verdade poderia ser fruto de diferenças na composição de características individuais. Os resultados mostraram que, mesmo controlando por características composicionais importantes, a variabilidade entre municípios permaneceu estatisticamente significante. Esse resultado difere dos resultados de Tellez et al ${ }^{19}$ (2006), onde as diferenças entre unidades de segundo nível perderam significância estatística após controlar variáveis individuais e ecológicas. Essa diferença de achados pode ser devida ao fato de que as unidades de segundo nível de Tellez et $\mathrm{al}^{19}$ (2006) foram bairros, enquanto que no presente estudo foram municípios. $\mathrm{O}$ percentual de variância atribuível ao segundo nível de análises foi de $14 \%$ no presente estudo, e 2,4\% para Tellez et $\mathrm{al}^{19}$ (2006) para os modelos vazios.

Ao comparar os $\mathrm{R}^{2}$ das variáveis dos serviços com as variáveis socioeconômicas, os valores foram $11 \%$ e $14 \%$ respectivamente. Esses valores são diferentes dos valores encontrados por Nadanovsky \& Sheiham ${ }^{14}$ (1995) que relataram 3\% e 65\% para essas variáveis, respectivamente. Tais diferenças, em parte, podem ser explicadas pelo tipo de desfecho, ecológico versus individual, mas principalmente pelo desfecho em si. É possível que os serviços tenham tido um efeito benéfico em curto prazo, mostrado na prevenção das cavidades de cárie no presente estudo, mas tenha desaparecido numa avaliação de mais longo prazo da saúde bucal, como foi o caso do CPOD no estudo de Nadanovsky \& Sheiham ${ }^{14}$ (1995). Diferenças na composição dos serviços prestados nos países desenvolvidos ocidentais nas décadas de 1970 e 1980 e no Brasil nos anos 2000-2002 não devem explicar essa diferença nos $\mathrm{R}^{2}$. Isso porque dentistas de países desenvolvidos nas décadas de 1970 e 1980 realizavam as mesmas atividades preventivas que os dentistas no Brasil em 2000-2002. Além disso, no Brasil, as atividades restauradoras também compunham uma parcela grande do total das atividades e foram in- 
cluídas nas análises. Portanto, apesar do presente estudo e do estudo de Nadanovsky \& Sheiham ${ }^{14}$ (1995) terem sido realizados em épocas e em países diferentes, os procedimentos odontológicos prestados parecem comparáveis (restaurações, extrações, profilaxia, aplicação de fluoreto e selantes).

Considerando-se que este foi um estudo observacional com dados secundários, citam-se as possíveis limitações em relação à qualidade dos dados utilizados. Para minimizar a possibilidade de erros de aferição dos desfechos, uma vez que não se pode assegurar uma boa calibração entre todos os examinadores, eles foram dicotomizados. É considerável a possibilidade de sub e super notificação de procedimentos, fato que acarretaria em taxas de cobertura populacional alteradas. No entanto, o objetivo não foi obter taxas válidas, mas relações válidas dessas taxas com outras variáveis. Se a sub ou supernotificação dos procedimentos não estiver associada com as prevalências dos desfechos, então as correlações encontradas foram válidas.

Sendo estudo seccional, a temporalidade dos fatos não pode ser assegurada, assim como a relação causal. Para lidar com esse problema, a atividade odontológica recente foi relacionada com um desfecho da cárie dentária que refletisse a experiência mais atual, i.e., o dente cariado não obturado. O foco nesse indicador foi justamente para tentar aproximar exposição e desfecho no tempo, dado que o dente obturado e o dente extraído podem com mais freqüência indicar a experiência de cárie passada, de mais longo prazo.

Em conclusão, o serviço público de odontologia no Rio Grande do Sul pode ter contribuído para a redução no número de cáries não restauradas em jovens de 15 a 19 anos entre os anos 2000 e 2003, porém não foi possível detectar influência desse serviço na experiência total da cárie (CPOD) nesses jovens. Pode-se supor que o serviço odontológico teve um lado benéfico, prevenindo a cárie, mas compensou esse efeito obturando e/ou extraindo dentes desnecessariamente. Além disso, grande parte da variabilidade na prevalência de cáries não restauradas entre municípios não foi atribuível exclusivamente aos serviços e permanece por ser explicada.

\section{AGRADECIMENTOS}

Aos dentistas Leonardo Pavianni e Helenita Correa Ely, representantes da Seção de Saúde Bucal do Departamento de Ações em Saúde da Secretaria de Saúde de Estado do Rio Grande do Sul, pela cessão do banco de dados. 


\section{REFERÊNCIAS}

1. Ahovuo-Saloranta A, Hiiri A, Nordblad A, Worthington $\mathrm{H}$, Makela M. Pit and fissure sealants for preventing dental decay in the permanent teeth of children and adolescents. Cochrane Database Syst Rev (online). 2004(3):CD001830.

2. Akerman M, Nadanovsky P. Avaliaçäo dos serviços de saúde: avaliar o quê? Cad Saude Publica. 1992;8(4):361-5.

3. Andrulis DP. Access to care is the centerpiece in the elimination of socioeconomic disparities in health. Ann Intern Med. 1998 Sep 1;129(5):412-6.

4. Baldani MH, Vasconcelos AG, Antunes JL. Associação do índice CPO-D com indicadores sócio-econômicos e de provisão de serviços odontológicos no Estado do Paraná, Brasil. Cad Saude Publica. 2004;20(1):143-52.

5. Caldas Junior AF, Silveira RC, Marcenes W. The impact of restorative treatment on tooth loss prevention. Pesqui Odontol Bras. 2003 AprJun;17(2):166-70.

6. Cunha-Cruz J, Nadanovsky P, Faerstein E, Lopes CS. Routine dental visits are associated with tooth retention in Brazilian adults: the Pro-Saude study. J Public Health Dent. 2004 Fall;64(4):216-22.

7. Davenport C, Elley K, Salas C, Taylor-Weetman CL, Fry-Smith A, Bryan S, et al. The clinical effectiveness and cost-effectiveness of routine dental checks: a systematic review and economic evaluation. Health Technol Assess. 2003;7(7):iii-v, 1-127.

8. Ekstrand KR, Christiansen ME, Qvist V. Influence of different variables on the inter-municipality variation in caries experience in Danish adolescents. Caries Res. 2003 Mar-Apr;37(2):130-41.

9. Goldstein H, Browne W, Rasbash J. Partitioning Variation in Multilevel Models. Understanding Statistics. 2002;1(4):223-31.

10. Handler A, Issel M, Turnock B. A conceptual framework to measure performance of the public health system. Am J Public Health. 2001 Aug;91(8):1235-9.

11. Locker D. Does dental care improve the oral health of older adults? Community Dent Health. 2001 Mar; 18(1):7-15.

12. Lucas SD, Portela MC, Mendonca LL. Variações no nível de cárie dentária entre crianças de 5 e 12 anos em Minas Gerais, Brasil. Cad Saude Publica. 2005;21(1):55-63.

13. Marinho VCC, Higgins JPT, Sheiham A, Logan S. Combinations of topical fluoride (toothpastes, mouthrinses, gels, varnishes) versus single topical fluoride for preventing dental caries in children and adolescents. Cochrane Database Syst Rev (online). 2004(1):CD002781.

14. Nadanovsky P, Sheiham A. Relative contribution of dental services to the changes in caries levels of 12-year-old children in 18 industrialized countries in the 1970s and early 1980s. Community Dent Oral Epidemiol. 1995 Dec;23(6):331-9.

15. Pincus T, Esther R, DeWalt DA, Callahan LF. Social conditions and self-management are more powerful determinants of health than access to care. Ann Intern Med. 1998 Sep 1;129(5):406-11.

16. Rychetnik L, Frommer M, Hawe P, Shiell A. Criteria for evaluating evidence on public health interventions. J Epidemiol Community Health. 2002 Feb;56(2):119-27.

17. Sheiham A. Impact of dental treatment on the incidence of dental caries in children and adults. Community Dent Oral Epidemiol. 1997 Feb;25(1): 104-12.

18. Snijders TAB, Bosker RJ. Multilevel analysis: an introduction to basic and advanced multilevel modeling. London: Sage Publications; 1999.

19. Tellez M, Sohn W, Burt BA, Ismail Al. Assessment of the relationship between neighborhood characteristics and dental caries severity among low-income AfricanAmericans: a multilevel approach. J Public Health Dent. 2006 Winter;66(1):30-6.

20. Tickle M, Milsom KM, King D, Blinkhorn AS. The influences on preventive care provided to children who frequently attend the UK General Dental Service. Br Dent J. 2003 Mar 22;194(6):329-32.

21. Victora CG, Habicht JP, Bryce J. Evidence-based public health: moving beyond randomized trials. $\mathrm{Am}$ J Public Health. 2004 Mar;94(3):400-5.

22. Watt R, Sheiham A. Inequalities in oral health: a review of the evidence and recommendations for action. Br Dent J. 1999 Jul 10;187(1):6-12.

RK Celeste foi apoiado pelo Conselho Nacional de Desenvolvimento Científico e Tecnológico (CNPq; bolsa de doutorado). 